\title{
Kidney Cancer: Current and Novel Treatment Options
}

Presented by Eric Jonasch, MD

\begin{abstract}
Antiangiogenic therapies remain the standard of care in the frontline setting for renal cell carcinoma, although vascular endothelial growth factor (VEGF) blockade is not sufficient, and many patients do not respond to such treatment. With a host of approved agents, questions arise as to how best to use them in both initial and secondary treatments. Optimal sequences are currently being tested in various clinical trials. Because approximately $20 \%$ of patients exhibiting primary resistance to these anti-VEGF therapies, new therapies are needed. Novel therapies such as MET and AXL inhibitors as well as checkpoint antibodies hold promise for the future. (J Natl Compr Canc Netw 2015;13:679-681)
\end{abstract}

"It has been an exciting 15 years for renal cell carcinoma," noted Eric Jonasch, MD, Associate Professor, Department of Genitourinary Medical Oncology, Division of Cancer Medicine, and Director of the Von Hippel-Lindau Clinical Center at The University of Texas MD Anderson Cancer Center. "When I started my career," he added, "we had 1 approved agent; now we have 8." Dr. Jonasch is Vice-Chair of the NCCN Guidelines Panels for Kidney and Testicular Cancers.

During his presentation at the NCCN 20th Annual Conference, Dr. Jonasch focused on the host of systemic therapies now available for metastatic renal cell carcinoma (RCC) that have category 1 evidence in the most recent version of the NCCN Guidelines. He stated that

\footnotetext{
Presented by Eric Jonasch, MD, Associate Professor, Department of Genitourinary Medical Oncology, Division of Cancer Medicine, and Director of the Von Hippel-Lindau Clinical Center at The University of Texas MD Anderson Cancer Center and co-Director of the MD Anderson Kidney Cancer Research Program, Houston, Texas. Dr. Jonasch has disclosed that he has received consulting fees or honoraria from AstraZeneca Pharmaceuticals LP, GlaxoSmithKline Novartis Pharmaceuticals Corporation, and Pfizer Inc., and grants or research support from Exelixis Inc., Novartis Pharmaceuticals Corporation, and Pfizer Inc.

Correspondence: Eric Jonasch, MD, The University of Texas MD Anderson Cancer Center, 1515 Holcombe Blvd, Unit 1374, Houston, TX 77030. E-mail: ejonasch@mdanderson.org
}

clinical study data do not support the use of adjuvant therapy for RCC in 2015. With the advent of various antiangiogenic agents and $\mathrm{mTOR}$ inhibitors, investigators have moved past the cytokine therapy era, he noted. In the upcoming years, Dr. Jonasch predicted, the next wave of clinical trials will test the combination of novel immunotherapies with antiangiogenic agents in the hope of further improving patient outcomes.

\section{First-Line Systemic Therapies for Metastatic Disease}

"Metastatic disease is treated in the appropriate situation (such as for a patient with a good performance status and a readily resectable primary tumor) with cytoreductive nephrectomy [Figure 1] and then systemic therapy [Figure 2]," stated Dr. Jonasch.

In the 2015 NCCN Guidelines for Kidney Cancer, first-line options with randomized phase II evidence of benefit include the FDA-approved agents sunitinib, pazopanib, and the combination of bevacizumab and interferon. Furthermore, temsirolimus is indicated for patients with a poor prognosis (category 1 evidence) but is a category $2 \mathrm{~B}$ option for select patients with other levels of risk.

Supporting phase III data behind the use of sunitinib in patients with metastatic RCC come from Motzer et al. ${ }^{1,2}$ In one trial, a significant improvement in progression-free survival (PFS) was seen with sunitinib over interferon-alfa (11 vs 5 months), ${ }^{1}$ with a borderline statistically significant benefit in overall survival despite robust crossover. ${ }^{2}$

The phase III data supporting the use of the oral agent pazopanib were reported by Steinberg et al, ${ }^{3}$ which compared pazopanib with placebo in treatment-naïve or cytokine-pretreated patients with advanced RCC. A 5-month increase in PFS was seen with pazopanib (9 vs 
Jonasch

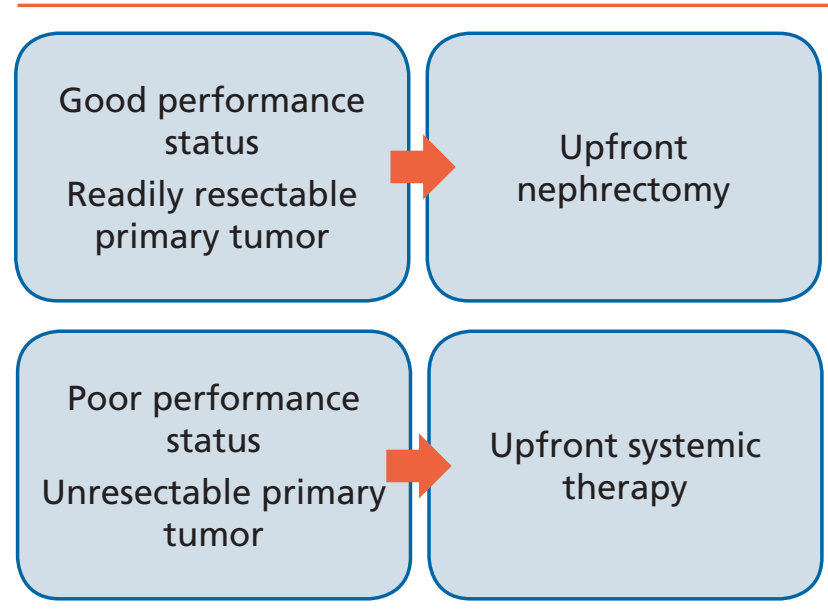

Figure 1 Upfront nephrectomy is still standard in appropriate patients.

4.2 months) in the overall population, and a nearly 8 -month increase (11 vs 2.8 months) was seen in the treatment-naïve population.

In the community-based phase III CALGB 90206 trial, the combination of bevacizumab and interferon-alfa was compared with interferon-alfa alone; median PFS was superior with combination bevacizumab and interferon-alfa. ${ }^{4}$ However, Dr. Jonasch noted that the modest difference in overall survival did not reach statistical significance.

Unlike the majority of studies with the other agents, early studies with temsirolimus suggested that it might be particularly helpful for those with more aggressive disease and poor-risk features. Poor-risk factors included lactate dehydrogenase level greater than 1.5 times the upper limit of normal, hemoglo-

- Stage 1, 2, 3:

- Nephrectomy

- Investigational question: adjuvant therapy?

- No role for targeted agents or IFN in this setting outside of a clinical trial.

- Stage 4:

- Cytoreductive nephrectomy for patients with performance status 0 or 1 , and resectable primary.

- Avoid doing nephrectomy on patients with high disease burden.

- Systemic therapy as per guidelines. bin less than the lower limit of normal, corrected calcium greater than $10 \mathrm{mg} / \mathrm{dL}$, time from diagnosis to first treatment of less than 1 year, Karnofsky performance status between 60 and 70, and multiple organ sites of metastasis. Phase III study data revealed a statistically significant improvement in median overall survival of 10.9 months with temsirolimus compared with interferon in this patient population. ${ }^{5}$

Finally, according to phase III data on sorafenib in pretreated patients with advanced RCC, ${ }^{6}$ a 5.5 -month PFS was seen with sorafenib versus 2.8 months for placebo.

"When this was presented, we were very happy with that," admitted Dr. Jonasch. "We have come some ways since then and now have higher expectations for treatments in RCC."

However, in a small phase II trial of first-line treatment with sorafenib versus interferon in RCC, no difference was seen in median PFS, according to Dr. Jonasch. "This dampened enthusiasm for sorafenib in the front-line setting," he added.

\section{Second-Line Treatments}

Dr. Jonasch called the second-line options for the most part "a not strongly evidence-based laundry list of remaining agents that are available." He focused his discussion on 2 particular agents with category 1 evidence: everolimus and axitinib. The phase III data on everolimus came from the RECORD- 1 trial. ${ }^{7}$ PFS rates favored everolimus over placebo (4.99 vs 1.67 months), with the overall survival curves "overlapping with crossover built into this trial," noted Dr. Jonasch.

The AXIS trial was the vehicle for the data supporting the use of axitinib over sorafenib in patients whose disease progressed after sunitinib, bevacizumab plus interferon-alfa, temsirolimus, or cytokine-based therapy. ${ }^{8}$ Even more interesting than the improvement in PFS with axitinib, added Dr. Jonasch, was the finding in the subgroup analysis by prior regimen.

With axitinib, PFS after cytokine therapy was 12.1 months versus 6.5 months with sorafenib. Dr. Jonasch called this "a pretty amazing number with axitinib." However, the benefit after the vascular endothelial growth factor (VEGFR) inhibitor sunitinib was 4.8 months with axitinib compared with 3.4 months with sorafenib, suggesting that using one VEGFR inhibitor after another may "burn out some of the benefit," he noted.

Figure 2 Treatment by stage. 


\section{Emerging Treatments on the Horizon}

Although antiangiogenic therapy in the frontline setting remains the standard of care, Dr. Jonasch admitted that anti-VEGF therapy is not a "magic bullet." VEGF blockade is important but certainly not sufficient, he added, opening the door for novel therapies in RCC. Among the more promising options, the focus is on new cancer cell or stromal targets through MET and AXL inhibition. Immune therapies, such as checkpoint antibodies, may also show promise. "We should be targeting the tumor cell itself, but at this point in time, we are sort of targeting the microenvironment," noted Dr. Jonasch.

Amplification of the MET gene has been identified in both papillary and clear cell RCCs, revealed Dr. Jonasch. In fact, high total MET expression has been linked to shorter PFS and overall survival in patients with metastatic RCC. ${ }^{9}$ The dual VEGFR2 and MET inhibitor foretinib has been studied in papillary RCC, ${ }^{10}$ yielding a 9.3-month PFS, indicating there may be some value in this approach. Phase II clinical trials of other MET inhibitors (AZC6094 and INC280) are ongoing.

Immune checkpoint antibodies have been "big news" in melanoma and lung cancer, and now appear to be promising in kidney cancer as well. ${ }^{11}$ One of these agents, nivolumab, yielded a modest improvement in PFS but a more impressive median overall survival of up to 25 months in patients with metastatic RCC, ${ }^{12}$ which Dr. Jonasch claimed is "outside the norm for second-line studies." In addition, ipili- mumab plus nivolumab and MK3475 in combination with other approved agents are the focus of ongoing clinical trials.

\section{References}

1. Motzer RJ, Huston TE, Tomczak P, et al. Sunitinib versus interferon alfa in metastatic renal-cell carcinoma. N Engl J Med 2007;356:115-124.

2. Motzer RJ, Hutson TE, Tomczak P, et al. Overall survival and updated results for sunitinib compared with interferon alfa in patients with metastatic renal cell carcinoma. J Clin Oncol 2009;27:3584-3590.

3. Steinberg CN, Davis ID, Mardiak J, et al. Pazopanib in locally advanced or metastatic renal cell carcinoma: results of a randomized phase III trial. J Clin Oncol 2010;28:1061-1068.

4. Rini BI, Halabi S, Rosenberg J, et al. Bevacizumab plus interferon-alpha versus interferon-alpha monotherapy in patients with metastatic renal cell carcinoma: results of overall survival for CALGB 90206 [abstract]. J Clin Oncol 2009;27(Suppl):Abstract LBA5019.

5. Hudes G, Carducci M, Tomczak P, et al. Temsirolimus, interferon alfa, or both for advanced renal-cell carcinoma. N Engl J Med 2007;356:22712281.

6. Escudier B, Eisen T, Stadler WM, et al. Sorafenib in advanced clear-cell renal-cell carcinoma. N Engl J Med 2007;356:125-134.

7. Kay A, Motzer R, Figlin R, et al. Updated data from a phase III randomized trial of everolimus (RAD001) versus placebo in metastatic renal cell carcinoma [abstract]. Presented at the 2009 ASCO Genitourinary Cancers Symposium; February 26-28, 2009; Orlando, FL. Abstract 278.

8. Rini BI, Escudier B, Tomczak P, et al. Comparative effectiveness of axitinib versus sorafenib in advanced renal cell carcinoma (AXIS): a randomized phase 3 trial. Lancet 2011;378:1931-1939.

9. Zhou L, Liu X, Sun M, et al. The mechanisms of anti-VEGF therapy resistance in renal cell carcinoma [abstract]. Cancer Res 2014;74:Abstract 687.

10. Choueiri TK, Vaishampayan U, Rosenberg JE, et al. Phase II and biomarker study of the dual MET/VEGFR2 inhibitor foretinib in patients with papillary renal cell carcinoma. J Clin Oncol 2013;31:181-186.

11. Postow MA, Callahan MK, Wolchok JD. Immune checkpoint blockade in cancer therapy [published online ahead of print January 20, 2015]. J Clin Oncol, pii: JCO.2014.59.4358.

12. Motzer RJ, Rini BI, McDermott DF, et al. Nivolumab for metastatic renal cell carcinoma results of a randomized phase II trial. J Clin Oncol 2015;33:1430-1437. 